\title{
Performance Analysis of a Wireless Sensor Network for Seism Detection in an Overlay Cognitive Radio System
}

\author{
Hassel Aurora Alcalá Garrido ${ }^{2}$, Mario Eduardo Rivero-Angeles ${ }^{1}$, Izlian Yolanda \\ Orea-Flores $^{2}$, Ramsés Rodríguez ${ }^{3}$ \\ ${ }^{1}$ Communication Networks Laboratory, CIC-IPN. \\ Av. Juan de Dios Bátiz, Col. Nueva Industrial Vallejo, Gustavo A. Madero, Mexico City, \\ 07738. \\ ${ }^{2}$ Telematics Department, UPIITA-IPN. \\ 2580 Instituto Politécnico Nacional Avenue, Barrio La Laguna Ticomán, Gustavo A. Madero, \\ Mexico City, 07340. \\ ${ }^{3}$ SEPI ESIA-IPN \\ Zacatenco. Luis Enrique Erro Avenue, Zacatenco, Gustavo A. Madero. Mexico City, 07738
}

\begin{abstract}
This paper studies the performance of a wireless sensor network (WSN) with cognitive radio (CR) capabilities to gather information about structural health monitoring (SHM) of buildings in case of seismic activity. The system performance is evaluated in terms of energy consumption and average packet delay using a discrete event simulator. In order to efficiently use the resources of the network and considering that the WSN will be installed in office or home buildings, we propose the use of the empty (white) spaces in a conventional cellular system. Thus, the WSN will act as secondary network, which do not affect the quality of service of the primary network, since it will work in an overlay structure. The main performance parameter of the primary network is the blocking probability since a Blocked Call Cleared (BCC) system is considered. By using the data collected in the WSN and transmitted through the unused channels of the cellular system, it is possible evaluate the damage caused by the seismic activity in a certain building. The major benefit of using a WSN with CR capabilities compared to the traditional approach, i.e., only one sensor per building floor or even per building is that with the WSN it is possible to collect much more information related to both the seism and the effects on the monitored building without the additional traffic in the already crowded unlicensed spectrum.
\end{abstract}

Keywords: Wireless Sensor Networks (WSN), Cognitive Radio (CR), Blocked Calls Cleared (BCC) system, Seism detection, Structural Health Monitoring (SHM)

\section{Introduction}

Wireless sensor networks (WSN) are composed of a large number of small sensor nodes with limited computing power, which are limited by their size and lifetime; usually they have a single omnidirectional antenna [1] to communicate with each 
other through wireless transmissions. The organization of their internal software and hardware must be configured properly in order to work effectively and be able to adapt dynamically to new environments, requirements and applications. Sensor networks must be able to adapt to different environmental changes in the monitored area, e.g. sensors may decrease their work cycles in order to reduce their power consumption when there is no significant change in general sensor readings [2].

Radio spectrum resources play a fundamental role in the wireless communication systems. The rapid growing demand for wireless communication services and some inefficient spectrum allocation methods result in the scarcity of the spectrum resources, which greatly hinders the development of future wireless communication systems. For instance, the fixed spectrum allocation approach ensures that wireless applications and devices do not cause any harmful interference with each other. However, it results in the inefficient use of the current radio spectrum. This results in scenarios where some bands are heavily occupied by busy radio services while other bands are seldom used at all. There are already great difficulties to find unassigned spectrum for the new broadband wireless communication services. One of the most promising solutions to overcome this problem is the cognitive radio (CR) technology. A CR device has the ability to identify an unoccupied spectrum band for temporarily usage, and vacate the spectrum when it is necessary. Therefore, CR is viewed as a technology to overcome the current inefficient usage of radio spectrum resources [3].

By dynamically changing its operating parameters, cognitive radio senses the spectrum, determines the vacant bands, and makes use of these available bands in a proper manner, improving the overall spectrum utilization. With these capabilities, cognitive radio can operate in licensed as well as unlicensed bands. In licensed bands wireless users with a specific license to communicate over the allocated band (the primary users, PUs), have the priority to access the channel. Cognitive radio users, also called secondary users (SUs), can access the channel as long as they do not cause interference to the PUs.

When a PU starts its communication, the cognitive radio user must detect the potentially vacant bands (spectrum sensing) and decide onto which channel to move (spectrum decision), finally adapt its transceiver so that the active communication is continued over the new channel (spectrum handoff). This sequence of operation outlines a typical cognitive cycle, which can also be applied over an unlicensed band by all cognitive radio users [4]. CR capabilities may also be exploited by WSNs, which are traditionally assumed to employ fixed spectrum allocation, and characterized by the communication and processing resource constraints of sensor nodes [5].

The BCC system is based on a TDMA (Time Division Multiple Access) system while the secondary network (the WSN) is a cluster based network that also uses a TDMA based protocol in the steady state (SS), considering that such protocol satisfies the functional requirements of the system by transmitting information in a collisionfree manner. Note that the use of a random access protocol could cause loss of packages for proper monitoring and timely analysis of seism related data. The system performance is studied using discrete event simulations performed in $\mathrm{C}++$.

This paper is organized as follows: First, Section 2, develops the design of an event-driven WSN applied to the early detection of damage in buildings in seismic areas with $\mathrm{CR}$ capabilities. The solution is aimed at improving the bandwidth 
utilization considering the cellular system as a primary network. Also, some relevant numerical results are presented. We present future work and some conclusions of our work in Section 3.

\section{System Operation}

In this section we present the design of the CR system where the cellular system is used as a primary and the secondary network is the WSN for seism detection and monitoring. The system is now described in detail.

\subsection{Secondary Network: WSN.}

The main variables that affect the performance of a WSN are: number of nodes in the network, number of Cluster Heads $(\mathrm{CH})$, transmission probability, coverage area, duration of steady state and time slot size.

The system operation begins by distributing the nodes in a random manner in the desired coverage area. Then, they are classified as either $\mathrm{CH}$ or Cluster Member $(\mathrm{CM})$. This is done by nodes transmitting a control packet to the entire network using the slotted NP-CSMA (Non-persistent Carrier Sense Multiple Access) protocol. The first $N$ nodes that successfully transmit their packet become $\mathrm{CH}$. The rest of the nodes also transmit their control packet in order to be associated as $\mathrm{CMs}$ to their closest $\mathrm{CH}$. At that time, all nodes are aware of their role in the network, either as $\mathrm{CH}$ or $\mathrm{CM}$, and $\mathrm{CMs}$ know at which $\mathrm{CH}$ they are associated to. Hence, each $\mathrm{CH}$ creates a routine time and assigns a time slot in a TDMA structure to each CM to transmit its information. In figure 1, we can see the basic system operation. It can be seen that there are two distinct phases: cluster formation and steady state. Note that in the cluster formation, transmissions are high energy demanding since all nodes have to transmit to the entire network while the steady state is a collision-free period where nodes report their reading to their respective $\mathrm{CH}$ and then the $\mathrm{CH}$ transmits the gathered data to the sink node.

To analyze the performance of the steady state phase, we consider an average of $M$ CMs per cluster, each transmitting packets of $P$ bits. If the total transmission rate is $R$ bits/sec, then the transmission time of the packet $T$ is $P / R$, this is taken as the duration of the slot. Hence, the frame duration is $M T$ [6].

It is important to notice that the $\mathrm{CH}$ consume much more energy than the $\mathrm{CM}$ simply because in the steady state $\mathrm{CHs}$ remain active at all times receiving data from the CMs and transmitting this data to the sink node. Again, the transmission to the sink is usually highly energetic since the sink node can be placed at a large distance from the network. Therefore, it is important to rotate the function of the $\mathrm{CH}$ to all nodes in the system. Building on this, it is considered that the steady state only lasts 20 seconds. After this time, clusters are broken in order to select new nodes as CHs.

To calculate the energy consumed in the WSN, the following values are proposed:

$E_{T x}^{C F}=1$ unit of energy used to transmit during the CF phase.

$E_{T x}^{S S}=0.5$ unit of energy to transmit from the $\mathrm{CM}$ to the $\mathrm{CH}$ at the steady state. 
$E_{T X}^{C H-B S}=1.5$ unit of energy consumed to transmit from the $\mathrm{CH}$ to the Base Station.

$E_{R x}=0.1$ unit of energy consumed for packet reception.

The rationale behind this is to consider general energy consumption and not to consider specific sensor nodes. Indeed, the actual energy consumption can be easily extended to any commercial node since the energy consumption is normalized at the value of $E_{T x}^{C F}$. The presented model attempts to capture the following behavior: a) when a packet is transmitted in the CF stage, nodes perform high-cost transmissions since all nodes in the network must follow the transmission of other nodes that are determining which cluster they will be assigned; b) while transmitting during the steady state phase, nodes already know to which $\mathrm{CH}$ they belong to. Since in average, $\mathrm{CM}$ are close to the corresponding $\mathrm{CH}$, these transmissions are less energetic; c) $\mathrm{CH}$ consume more energy to transmit the gathered information to the sink node since typically, the sink node is found outside the network area; d) packet reception consumes much less energy than packet transmissions.

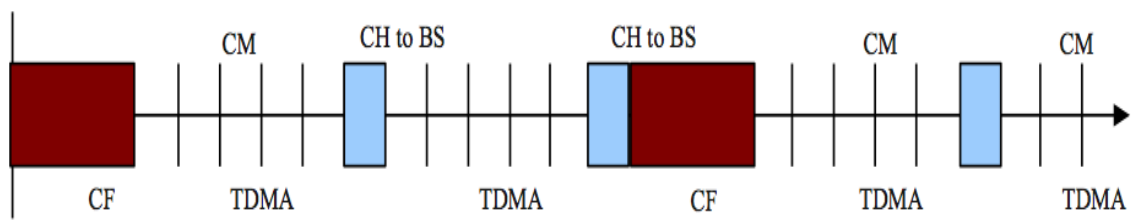

Fig. 1. Basic operation of the secondary network (WSN)

It is assumed that the length of the message of the structural health monitoring is 1 Kbit; taking into account the standard values for commercial devices, the value of 11 Mbps is taken as the baud rate of the wireless node. From this, it is easy to see that the size of the slot required to transmit the packet in the secondary network is 0.1 milliseconds.

\subsection{Primary Network: Cellular System}

In the case of the primary network, a simple BCC system is considered. Hence, nodes that arrive to the system and find no available resources are blocked. The variables considered are: arrival rate, average service time, number of servers, frame duration, and slot duration.

We consider an infinite population model, i.e. the traffic intensity remains unchanged regardless of the number of users being served in the system. In particular, we assume that the system can have a maximum of $N$ clients with an active call and if a new customer arrives when all servers are busy, the customers are blocked, i.e., it is denied their access to the system and they leave without being served [7]. Users arrive to the system according to a Poisson process, but only those who find available channels may be served.

In particular, we consider a TDMA based system such as GSM, GPRS or EDGE, which is composed of $N$ slots per frame to serve users. When a user arrives, it is 
assigned a slot and it will use it until the active call ends.

In Fig. 2, we can see an example of the allocation of the slots. At the beginning, the first and second frames, all time slots are busy. Thus, if a user requests to be served by the system, it will be rejected. In the third frame, slot 2 is free and can be used by a user who asks to be served by the system. In the last frame, a new user has occupied slot 2 again and slot 4 is now free.

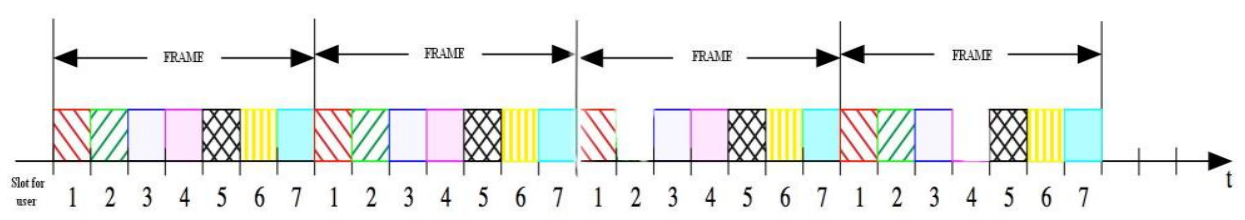

Fig. 2. TDMA in BCC System

In a GSM system, the frame is divided into eight time slots; the duration of a frame is 125 milliseconds and the size of the slot is approximately 15.625 milliseconds. Each user making a call is assigned a specific time slot for transmission, which enables multiple users to use the same channel simultaneously without interfering with each other.

Blocking probability is analyzed when the network operates with the TDMA scheme and it is compared to the Erlang B blocking formula for an offered traffic load in the range of 0.1 to 1 .

\subsection{Numerical Results}

In this section, the system is evaluated in terms of the average cluster formation time for the secondary network and blocking probability for the primary network. To this end, the following system parameters are considered: The number of nodes in the network ranges from 10 to 100 and are distributed in an area of 400 square meters (20m x 20m which corresponds to a typical building floor area in Mexico City). The number of $\mathrm{CHs}$ varies in the range of $10 \%$ to $50 \%$ of the nodes in the system. For example, if we have 100 nodes in the network, in the simulation we analyzed the case where there are 10, 20, 30, 40, and $50 \mathrm{CH}$.

Fig. 3 shows the average cluster formation time for different number of nodes in the network. It can be seen that with a transmission probability of 0.001 there is a large number of collisions with few nodes in the network, but as the number of nodes increases, also the collision probability slightly increases. For the case of the transmission probability of 0.1 for a small number of nodes there are few collisions, as the number of nodes in the network increases, the cluster formation time also increases due to the large number of collisions. Analyzing the value of 0.01 , we note that the number of collisions is small when we have few nodes and when we increase the number of nodes there is not an abrupt increase in the graph, also the collision probability slightly increases. Therefore, 0.01 can be an appropriate value for the considered environment. 


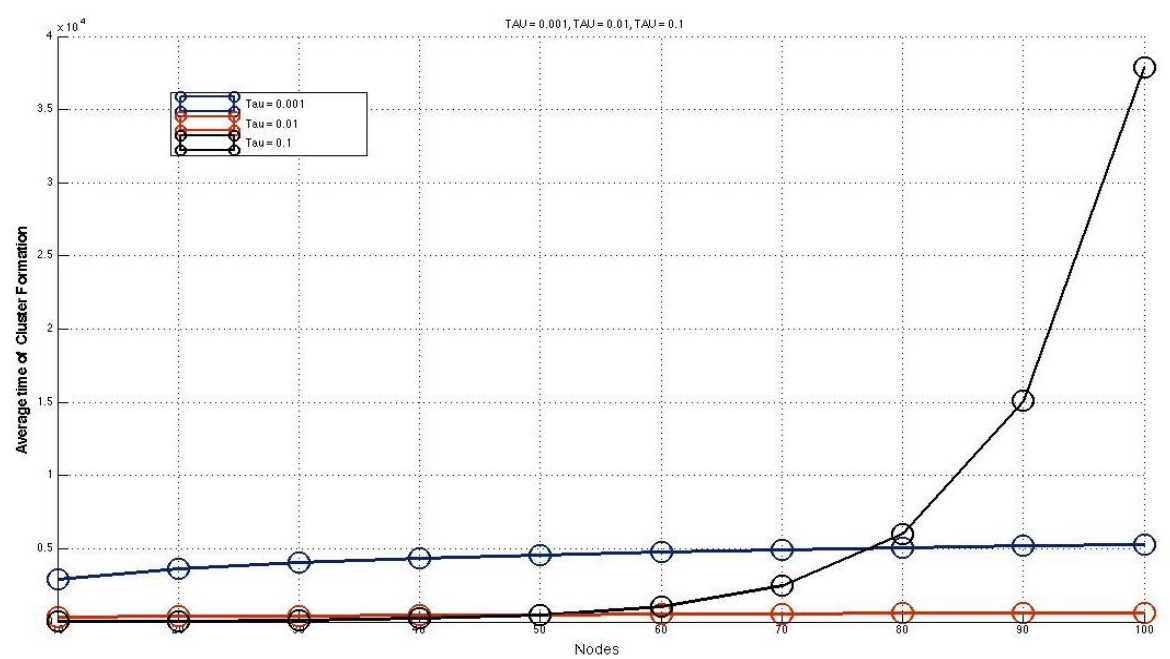

Fig. 3. Average cluster formation time with transmission probability $0.001,0.01$ and 0.1 .

In Fig. 4, the blocking probability of the primary network for the simple BCC system is presented. If we have a few servers, the blocking probability is high. As the number of servers increases, the blocking probability decreases.

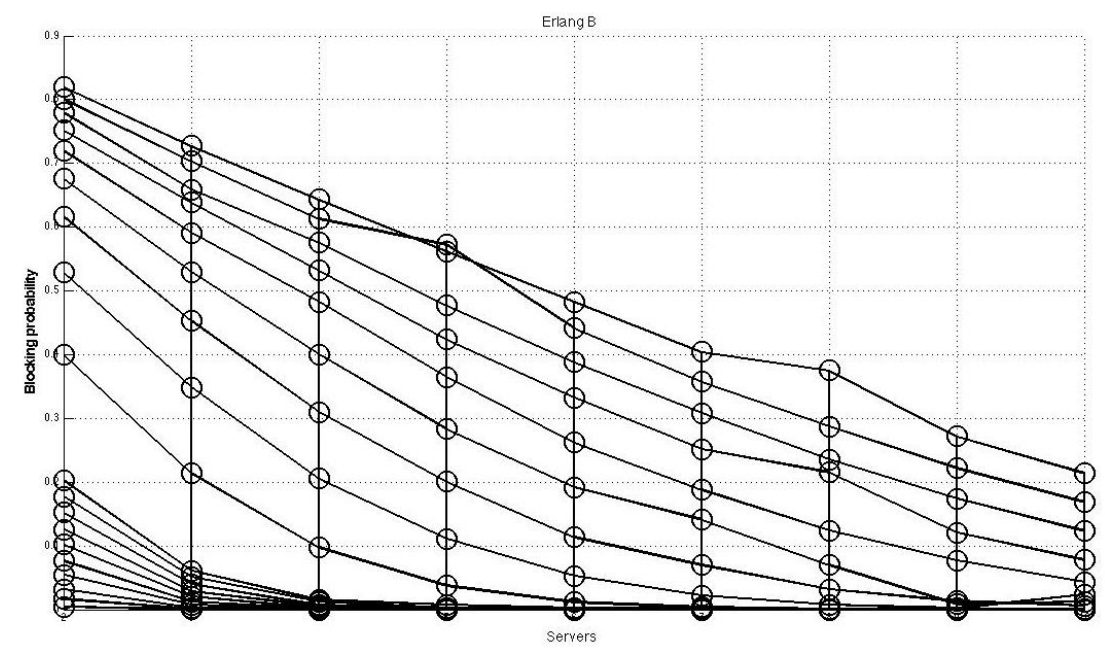

Fig. 4. Blocking probability of primary TDMA network without regarding to the number of servers.

In Fig. 5, we can notice the blocking probability relative to the offered traffic. When there is a small traffic load on the network, the blocking probability is small; while a very large offered traffic entails a high blocking probability. 


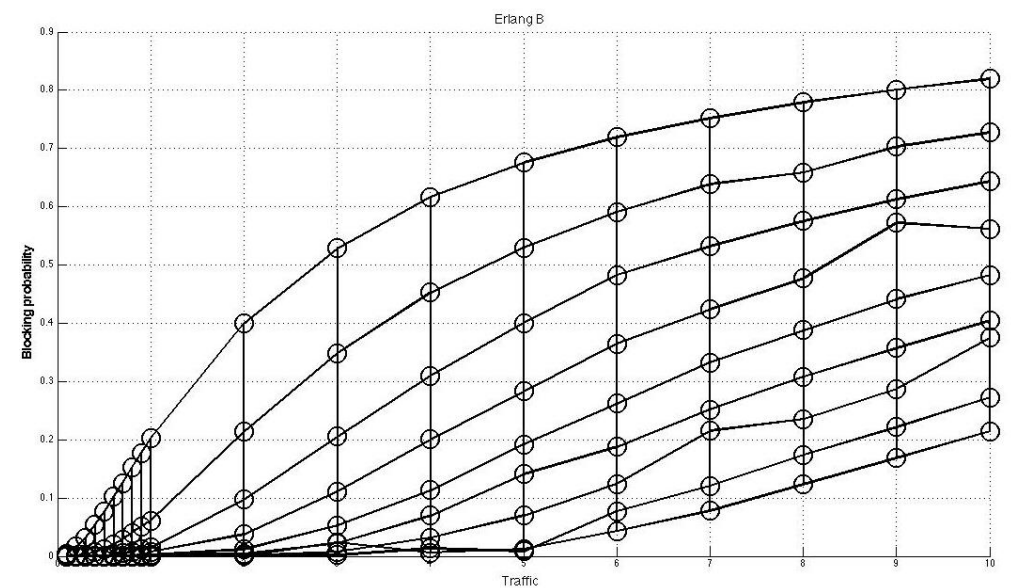

Fig. 5. Blocking probability of primary TDMA network without regarding to the offered traffic.

Finally, in Fig.6, we can see a comparison of the blocking probability in the primary network for the simple BCC system and with the TDMA structure for 8 time slots with the traffic load in the range of 0.1 to 1 . In general, it can be seen that there is a good match between those results with a slight difference for high offered loads.

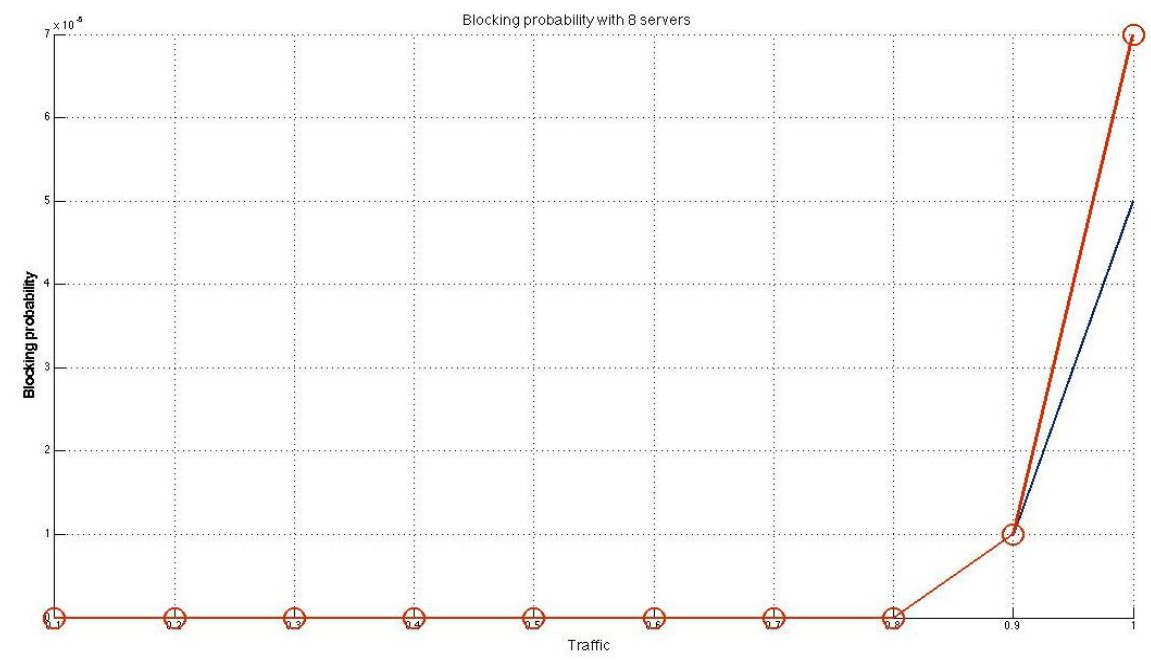

Fig. 6. Comparison of the blocking probability in the primary network with and without the TDMA structure.

\section{Conclusions and future work}

A WSN is proposed to be monitoring seismic activity and for transmission of relevant data in case of telluric movements in buildings by making use of the white spaces from a cellular system under a cognitive radio network. 
We note that a cellular system as a primary network is suitable for the transmission of data to the secondary network since the blocking probability can be lower than $5 \%$ while maintaining empty spaces in the TDMA frame.

Furthermore, in our future work, we plan to model the behavior of the primary and secondary network mathematically and verify analytical results with simulation results.

\section{References}

1. Hu, X., Wang, B., \& Ji, H. (2013). "A Wireless Sensor Network-Based Structural Health Monitoring System for Highway Bridges". Computer-Aided Civil and Infrastructure Engineering, Pp. 193-209.

2. Pradnya, G. \& Anjali, M. (2008). "A survey of architecture and node deployment in Wireless Sensor Network”. IEEE. Pp. 426-430.

3. Xue, J., Feng, Z. \& Chen, K. (2013). "Beijing Spectrum Survey for Cognitive Radio Applications" . Beijing Univesity of Posts and Telecommunications. Beijing: IEEE.

4. Akan, B., Karli, O. \& Ergul, O. (2009). “Cognitive Radio Sensor Network”. IEEE Network.

5. Reddy, V., Krisha R. \& Reddy, M. (2011). "Sensor Networks for Cognitive Radio: Theory and System Design". IEEE.

6. Rom, R. \& Sidi, M. (1989). "Multiple Access Protocols. Performance and analysis”. Haifa, Israel: Springer-Verlag. Pp. 1-7.

7. Kleinrock, Leonard. (1975). “Queueing systems”. John Wiley \& Sons, Inc. Pp. 103 